\title{
Zur „Berichtigung“" Dr. Glasscheibs zu meinem Artikel: Schädigung der Zähne nach Radikaloperation von der Oberkieferhöhle.
}

\author{
Von \\ Dr. Halle. \\ (Eingegangen am 3. Mai 1931.)
}

Herr Glasscheib schreibt, daß er die Oberkieferhöhlenoperation in der jetzt von mir dargestellten und begründeten Form nicht von mir ausgeführt gesehen habe. Das mag zutreffen. Aber er unterläßt es zuzugestehen, daß ich bei der Ausführung meiner Ozaenaoperation vor meinen Assistenten und Kursteilnehmern immer wieder darauf hingewiesen habe, daß sie sich ausgezeichnet auch für die Radikaloperation der Oberkieferhöhlenempyeme eignet, weil man durch die temporäre Verlagerung der medialen Kieferhöhlenwand einen überraschend guten Ưberblick über die Höhle bekommt und mit großer Sicherheit alle Buchten und Nischen der Höhle erreichen kann. Daß diese häufigen Darlegungen oft in Gegenwart des Herm Glasscheib erfolgt sind, wird er schwerlich abstreiten wollen. Es wären auch dafür genügend unverdächtige Zeugen vorhanden.

Herr Glasscheib. wird nicht in Abrede stellen wollen, daß wir nicht selten Fälle von Ozaena, die mit chronischen Empyemen kompliziert waren, mit bestem Dauererfolge operiert haben. Dabei ist es an sich gleichgültig, ob man die Höhle für kurze Zeit vorn offen hält, entsprechend dem perpendikulären Schnitt bei der Ozaenaoperation, oder ob man, wie ich es beschrieben habe, die natürliche Öffnung mittels Bougies oder dergleichen genügend erweitert, um bequem von dort aus nachbehandeln zu können, oder endlich, ob man sich entscheidet, ein Verfahren anzuwenden, das wir seit Jahrzehnten bei der Radikaloperation der Kieferhöhle gebrauchen. Die Bildung des Schleimhautlappens im unteren Nasengang, die zuerst Boenninghaus als Hineinstoßen der Schleimhaut in die Höhle in etwa sternartiger Form beschrieben hat, während Dahmer dem Lappen zuerst die heute übliche sorgsam geschnittene Form gab, und der daher wohl richtig der Boenninghaus-Dahmersche Lappen heißt - die Bildung dieses Lappens bei jeder Art von Radikaloperation des Antrums ist längst Allgemeingut jedes Operateurs auf diesem Gebiete. Und wenn Glasscheib emphatisch betont, die Kombination der von mir zugegebenermaßen erlernten Ozaenaoperation 
und ihrer Verwendung als Radikaloperation der Kieferhöhle, die er leider verschwiegen hat, mit dem Boenninghaussehen Lappenschnitt sei seine „ureigenste Erfindung", so ist das zwar ein bißchen wenig. Aber man könnte ihm diese „Erfindung" gern zugestehen, wenn es jemand gibt, der diese Leistung überhaupt als Erfindung gelten lassen wollte. Aber dann fehlte eben seitens des Herrn Glasscheib der Hinweis nicht nur auf meine Oza.enaoperation, die ich schon publiziert hatte, sondern auch auf meine ihm sozusagen vertraulich mitgeteilten und ihm wohlbekannten Darlegungen hinsichtlich ihrer Verwendung zur Radikaloperation der Kieferhöhle, deren Indikation ich nur noch vor der Publikation an meinem großen diesbezüglichen Material hinreichend erproben wollte, was Herr Glasscheib augenscheinlich nicht so nötig hatte, da er seine „Erfindung“ auffallend kurze Zeit nach dem Scheiden aus meinen Instituten veröffentlicht hat.

Ich habe zwar weder von dieser Arbeit des Herrn Glasscheib noch von seinen späteren Veröffentlichungen einen Separatabdruck bekommen, habe ihn aber gelesen. Von der schon damals beabsichtigten Entgegnung, die immer recht wenig erfreulich werden mußte, da ich Herrn Glasscheib in diesem Falle keinen guten Glauben hätte zugestehen können, glaubte ich absehen zu sollen, da in derselben Zeitschrift 1924 eine Entgegnung von Dahmer erschien, die sich gegen Herrn Glasscheib wandte. Mir kam es in erster Linie auf die Sache an, und darum wollte ioh erproben, ob man diese Methode als Regel empfehlen dürfte, und warum die bisherigen Methoden weniger zweckmäßig wären.

Ich hatte schon an der Lautenschlägerschen Operation der Ozaena, bei aller Anerkennung ihrer neuartigen Idee, auszusetzen gehabt, daß sie auf beiden Seiten große Schleimhautschnitte im Munde, eine umfangreiche Fortnahme der Fossa canina und eine eventuelle Schleimhautplastik verlangte. Daher die Ausbildung meiner intranasalen Operation, die zwar die Idee der Lautenschlägerschen aufnahm, aber ungemein viel konservativer ist, so gut wie keine Zerstörungen macht und nach meinen Erfahrungen bei guter Ausführung mindestens die gleichen guten Resultate zeitigt. Die Schonung von Schleimhaut und Knochen stand auch bei der Anwendung meiner Ozeanæoperation für die Radikaloperation der Oberkieferhöhle an erster Stelle, abgesehen von den sonstigen Annehmlichkeiten für den Patienten.

Nun belehrt mich Herr Glasscheib, daß die Zahnnerven gar nicht durch meine Methode geschont werden, da sie am Boden der Kieferhöhle liegen. Es werde nur die Parästhesie und Anästhesie der Lippen vermieden. Nun das wäre ja auch schon ein nicht unwichtiger Vorteil. Aber ich habe doch lieber noch einmal in den mir zugänglichen Lehrbüchern der Anatomie nachgeschlagen. Und da fand ich übereinstimmend: Bricht man die obere Wand des Canalis infraorbitalis weg, so findet man an der unteren Wand desselbèn, von außen nicht sichtbar, feine Öffnungen, 
die Foramina alveolaria superiora, anteriora und media, in welche die gleichnamigen Zweige des N., der A. und V. infraorbitalis hineintreten, um in kleinen Knochenkanälchen zu den Wurzeln der vorderen und mittleren Zähne zu verlaufen.

Und an einer anderen Stelle: Die Nn. alveolares superiores, medii und anteriores verlaufen in den nach ihnen benannten Kanälchen des Oberkieferbeins bis zu den Zahnwurzeln, die sie in Form eines Geflechtes, des sog. Plexus dentalis superior, umspinnen. Von diesem Plexus treten die oberen Zahnnerven in die Zahnwurzeln usw. Außerdem gehen vom $\mathrm{N}$. infraorbitalis feine Rami gingivales und buccales, Rami faciales usw. $\mathrm{ab}$ und auch Äste für die Schleimhaut der Oberkieferhöhle. -

Die Anatomen scheinen danach doch anderer Ansicht zu sein als Herr Glasscheib, und er wird es mir nicht übel nehmen, wenn ich ihrer Autorität eher folge. Dann aber müssen durch die oft weitgehende Fortnahme der Fossa canina usw. die Nerven und Gefäße der Zähne in großem. Umfange zerstört werden, und die Folge ist die nicht selten so sehr beklagte Parästhesie und. Anästhesie der Zähne und der Wange.

Nun schreibt Herr Glasscheib: Er (Halle) will genau so mit meiner Arbeit verfahren wie mit der Tränensackoperation von West !!! Herr Glasscheib! Wenn andere Kollegen aus Unkenntnis der Literatur auch die Operation nach West benennen konnten, Sie durften das nicht. Sie durften auch einen derartigen Anwurf gegen mich nicht erheben. Sie waren durch meine zahlreichen Darlegungen genauestens über die Tatsachen informiert, die zu besprechen oder zu widerlegen Thnen wie jedem meiner Assistenten jederzeit offen stand. Sie mußten die Daten kennen, die ich zuletzt noch in Basel genauestens publiziert habe, oder Sie mußten sie richtigstellen. Herr West hat das Verdienst, die von ihm so genannte ,Fensterresektion des Ductus" geschaffen zu haben, die er am 14. 10. 1910 in der Berl. lar. Ges. vortrug. Er hat mich dadurch zu meiner Tränensackoperation mit Bildung eines gefensterten Schleimhautperiostlappens angeregt, wie ich immer betont habe. Einen derartig operierten Fall stellte ich am 12. 5. 1911 in derselben Gesellschaft vor.' Ich beschrieb damals schon alle Einzelheiten, wie ich sie später genauer veröffentlicht habe. West konnte daran keinen Anteil haben, da er unmittelbar nach seinem Vortrag in der Berl. lar. Ges. nach Amerika zurückkehrte. Als er wieder nach Berlin kam, hat er seine ersten 100 Fälle in meiner Klinik operiert. Er hat bei mir die Abänderung und Erweiterung seiner Methode gesehen und begründet bekommen, da ich in weitestgehender Kollegialität gewohnt war, alles zu zeigen, was bei mir geschah. Er hat, wie Herr Glasscheib leicht hätte in den Protokollen nachlesen können, in meiner Gegenwart in der Berl. lar. Ges. mein Verfahren für gut erklärt, hat auch bei seinen späteren Publikationen etwa 2 Jahre danach (!) nur meinen Lappen verändert und verschlechtert. Polyak selber hat anerkannt, daß die erste Eröffnung des Sackes mit Lappenbildung von 
mir stammt, anerkannte Autoren, wie Herzog, haben es bestätigt. Aber wenige Kollegen gaben sich die Mühe, sich von den Tatsachen zu überzeugen, die eigentlich deutlich genug durch die Protokolle der Berl. lar. Ges. festgelegt sind. Herr Glasscheib aber jedoch unterrichtet, mußte sich zumindest unterrichten, wie die Dinge sich unbezweifelbar verhalten.

Aber damit er ein andermal noch mehr Material gegen mich hat, will ich gleich einen anderen Fall hinzufïgen, wo meine weitgehende Kollegialität mir ebenfalls üblen Dank brachte. Ich habe eine neue Methode der Gaumenoperation angegeben, die auch bei Erwachsenen beste klinische und funktionelle Erfolge ermöglicht. Ich habe bei meiner diesbezüglichen Publikation auf ähnliche Bemühungen Ganzers hingewiesen und habe berichtet, daß ich zu dieser Operation durch Dr. Ernst vom Zahnärztlichen Institut in Berlin angeregt worden bin, der mir an Patienten seine Prothesen für Gaumendefekte zeigte und mir sagte, die bisherigen Gaumenoperationen hätten, zumal bei Erwachsenen, wenig Zweck, weil die Sprache danach wenig oder keine Besserung zeigte. Die Erfolge könnten erst besser werden, wenn man eine Annäherung des Gaumens an die Rachenwand ermöglichen könnte. Und er meinte damals, daß es meiner von ihm sehr anerkannten Technik gelingen müßte, eine solche Operation zu finden. Nun es gelang mir, und ich habe sie auf dem Kongreß unserer Gesellschaft vorgetragen, habe in der Berl. lar. Ges. und in der Berl. med. Ges. darüber gesprochen und Patienten vorgestellt, die allgemein anerkannte Resultate zeigten. Herr Ernst hat vom Jahre 1915-21 auf seinen Wunsch fast allen derartigen Operationen als Zuschauer beigewohnt. Er hat mir aber weder einen Patienten übergeben, noch je irgend einen Hinweis über die mögliche Technik gemacht, wozu er um so weniger qualifiziert war, als er vorher niemals ein Messer in der Hand gehabt hatte. Aber im Jahre 1921 stellte er in der Chir. Ges. die Methode als die seinige vor, ohne auch nur meinen Namen zu nennen. Und nach einem Versprechen mir gegenüber, das richtigzustellen, veröffentlichte er später in einer zahnärztlichen Zeitschrift: Zur Ausführung dieser Idee gewann ich zunächst Halle-Berlin. Das war alles. Ich habe zwar Herrn Ernst vor dem Forum der Berl. med. Ges. zur Rede gestellt und ihm diese Tatsachen vorgehalten. Aber ich bin nicht gewiß, daß er, mit minderem Rechte als Herr West, nicht weiterhin die Methode als die seinige erklärt, wenn auch Limberg und Rosenthal u, a. es korrekt dargestellt haben. 